\title{
Ascension and Port Stanley geomagnetic observatories and monitoring the South Atlantic Anomaly
}

\author{
Susan Macmillan, Chris Turbitt and Alan Thomson \\ British Geological Survey, Edinburgh, United Kingdom
}

\begin{abstract}
Our 15-year experience of operating two remote observatories, Ascension and Port Stanley, in the south Atlantic is described. These observatories help monitor the South Atlantic Anomaly (SAA), a region of weak magnetic field which causes considerable problems for spacecraft operators. One-minute and one-second values from these observatories, and other observatories both inside and outside the SAA, are analysed. We investigate whether the SAA, and its growth over time, are having any tangible effect on the observed external field variations. Whilst only able to illustrate the long-term characteristics of the irregular external field related to the solar cycle and not due to any long-term changes in the internal field, we do isolate micropulsation signals at sites inside the SAA which contain more power than at sites outside.
\end{abstract}

Key words geomagnetism, observatories - South Atlantic Anomaly - secular variation - micropulsations

\section{Introduction}

Since the early 1990s the British Geological Survey has operated two observatories, Ascension and Port Stanley, in the south Atlantic and as a result has produced near-homogeneous 1minute mean absolute geomagnetic datasets which span about 15 years. Along with datasets from several other observatories, this provides an excellent resource for understanding the electromagnetic environment in this region of the world. One feature of particular interest is the South Atlantic Anomaly (SAA), an area where the radiation hazard from high proton fluxes and cosmic rays is high due to the rela-

Mailing address: Dr. Susan Macmillan, British Geological Survey, Murchison House, West Mains Road, Edinburgh, EH10 4AZ, UK; e-mail: smac@bgs.ac.uk. tively low shielding effect provided by the Earth's magnetic field. In this area, for example, a significantly higher number of so-called single event upsets (SEUs) occur in low earthorbiting satellites (Heirtzler, 2002). This area is related to a region of reverse flux at the core mantle boundary beneath the south Atlantic where the magnetic field is directed into the core rather than out, as it is elsewhere in the southern hemisphere (Gubbins and Bloxham, 1985). The apparent growth of this reversed flux region over the last hundred years or so (Jackson et al, 2000) may be related to the Earth's magnetic field attempting to reverse (Gubbins, 2008).

In this paper we describe our experience of operating Ascension and Port Stanley observatories and the difficulties encountered in doing this remotely from the UK. Using data from these observatories and others in the area which have been running for several decades, and repeat station observations, we then look at how the SAA is evolving in space and in time.

We then investigate the hypothesis that the SAA, and any long-term changes in it, have an 
impact on the processes which generate external field variations. First, we investigate whether the growth of the reverse flux patch at the core-mantle boundary in the south Atlantic influences the irregular external magnetic fields observed in this part of the world (as opposed to the regular diurnal variation). We describe an analysis involving hourly standard deviations computed from one-minute data recorded at a number of observatories operating inside and outside the SAA area. We then look at the high frequency end of the spectrum, analysing pulsation signals from observatories inside and outside the SAA.

\section{Ascension and Port Stanley operational experiences}

The British Geological Survey (BGS) has operated magnetic observatories on Ascension Island (ASC) and the Falkland Islands (PST) since 1992 and 1994 respectively. These two remote South Atlantic locations were selected to increase the global distribution of continuous, quality vector magnetic data.

Unlike BGS observatories in the United Kingdom, it is not economically feasible to maintain the South Atlantic observatories as permanently manned stations. Operating an unmanned observatory demands that the instruments, recording facilities and data communications are reliable and cost-effective. In addition to instrumental complications, the remoteness and climatic conditions at the sites have given rise to a variety of operating problems, adversely affecting the recorded data.

Trained local staff oversee the routine operation of both of the BGS South Atlantic observatories and perform a fortnightly (PST) or monthly (ASC) set of absolute observations. Since neither observatory is permanently manned, the selection of a suitable site was heavily dependent on the available infrastructure. As well as cost considerations, sites have been selected based on the requirement for a reliable power source, available data communications and ease of access. In general, these criteria are in competition with the requirement to locate at a magnetically clean site, free from

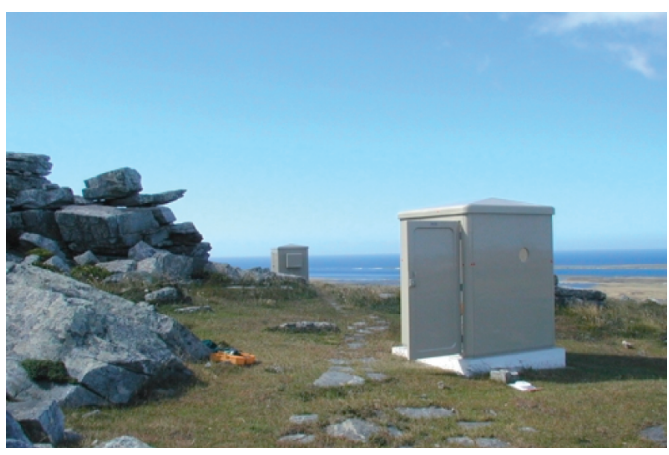

Fig. 1. The huts housing the magnetometers at Port Stanley.

potential man-made magnetic interference and, therefore, sites have been selected as a compromise between the two. The huts housing the magnetometers at PST are shown in fig. 1.

Since neither observatory is located on land owned or controlled by BGS, variometer data have frequently been contaminated by cultural noise. Often this noise is transient (temporary equipment or vehicles on site), but there have been instances of permanent interference, such as the installation of a steel mast at PST in June 1999. By operating both a fluxgate variometer and a proton precession magnetometer separated by a few metres, such interference can often be identified. At both observatories the absolute pillar is sited more remotely than the variometer (since electrical power is not required at the absolute pillar) and cultural noise can often be removed during post-processing of the variometer baselines. However, both observatories are located close to telecommunication installations and the instruments are intermittently subject to RF interference which can be less predictable in its effect and more difficult to remove. Some measures to reduce the effect of $\mathrm{RF}$ interference have had a degree of success, including shielding instruments and minimising cable lengths.

The logistics of operating a station remotely also have an effect on the continuity and quality of the data. Whilst absolute observations are made at least monthly, observatory visits by BGS staff for repairs, maintenance and 
observer training are normally restricted to once per year. The cost of transporting equipment to both sites is also restrictive - heavy items must be shipped by sea, a process which can take in excess of a month. As a consequence, instrument failures can lead to prolonged periods of missing data.

The upgrade of the communication link to both observatories in 2002 from a uni-directional satellite link to a bi-directional internet link provided a more robust, real-time means of monitoring the instrumentation, allowing faults to be diagnosed and often resolved remotely. This led to a significant improvement in the reliability of the data.

Prior to 2007, the fluxgate magnetometers, although insulated, were not maintained in a temperature stabilised environment. Although the insulation attenuated the diurnal temperature variations, the instruments were subject to seasonal temperature fluctuations, with a detrimental effect on the ability to maintain accurate baselines. The effect of external temperature variations on instrument baselines is more prominent in the temperate Falkland Island climate, rather than on the tropical Ascension Island. This problem is exacerbated at PST by the low frequency of absolute observations in the winter months due to the low temperature, strong winds and frequent precipitation. Therefore, temperature control (consisting of additional insulation, a $150 \mathrm{~W}$ non-magnetic heater and a proportional temperature controller) was added to the PST instrument enclosure in May 2007. The standard deviation in instrument temperature between May-December 2007 has been improved to $0.3^{\circ} \mathrm{C}$ compared to $4.0^{\circ} \mathrm{C}$ for the same period in 2006.

The frequency of absolute observations from PST has been improved since 2003 with the erection of a rudimentary fibre-glass enclosure, although the observatory often suffers from poor visibility affecting sighting the fixed mark.

The Falkland Islands also has a greater incidence of lightning strikes than Ascension Island and lightning has resulted in long periods of data loss on a number of occasions. In all cases, damage to electronic components has been caused by induced, transient currents in long data and power cables between the recording hut and the sensor hut, despite the inclusion of lightning protection barriers. It may be that the addition of large steel masts in close proximity to the instruments has increased the incidence of lightning strikes in recent years. Lightning induced failures have been eliminated since 2005 when all copper data lines were replaced with fibre-optic ones and the protection provided by the suppressors on the power lines was improved through better earthing.

Ascension Island, like many sites in the South Atlantic, is volcanic and magnetically anomalous - the observatory site has gradients in excess of $10 \mathrm{nT} / \mathrm{m}$ in parts. Although not evident in the ASC data, such localised magnetic signals can vary with time, temperature and perturbations such as lightning strikes. In high gradient fields, care must be taken to accurately locate absolute observing instruments, to avoid disturbing magnetised material (rocks) and to routinely survey the field intensity around the site. At very high gradient sites, it no longer becomes feasible to operate a vector observatory as the assumption that the field vectors at the variometer and absolute pillar are parallel is no longer valid. Consequently, it is not possible to apply baselines to correct the variometer data to the absolute pillar.

\section{Analysis of geomagnetic data}

The strength of the internal magnetic field is significantly lower in the south Atlantic compared to elsewhere in the world. This is shown in fig. 2 and it can be seen that the SAA is moving westwards and is deepening. From fig. 3 it can be seen that, as a percentage of the full field, the rate of change is also quite different in this part of the world. Both the magnitude of the conductivity perpendicular to the magnetic field and the mean height of the ionospheric dynamo layer are affected by the field strength, but how this rapid change in an already weak field affects the numbers of SEUs is not yet known.

Figure 4 shows the locations of the observatories and other sites where measurements of the full magnetic field vector have been made. 


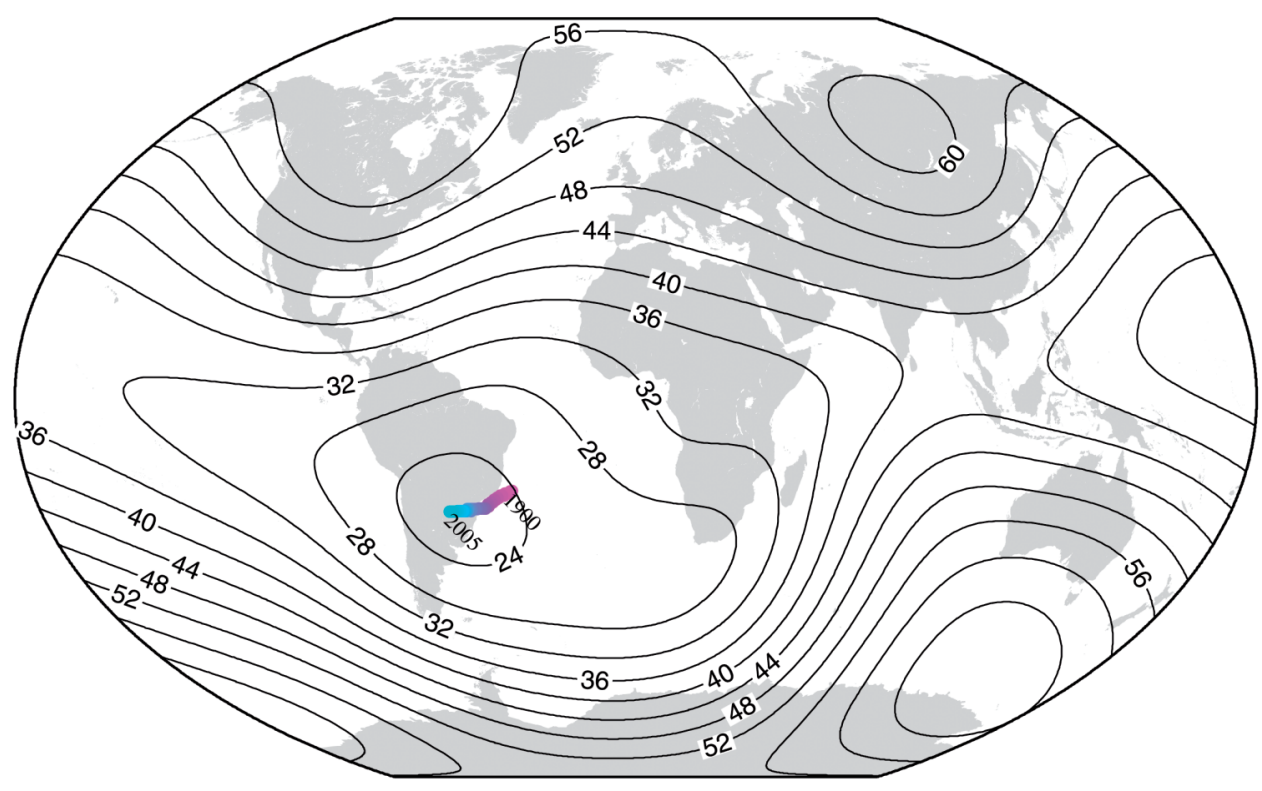

Fig. 2. Magnetic field strength $(\mu \mathrm{T})$ at 2005.0 and the loci of minima from 1900 from 10th Generation International Geomagnetic Reference Field (IGRF-10) (Maus et al., 2005).

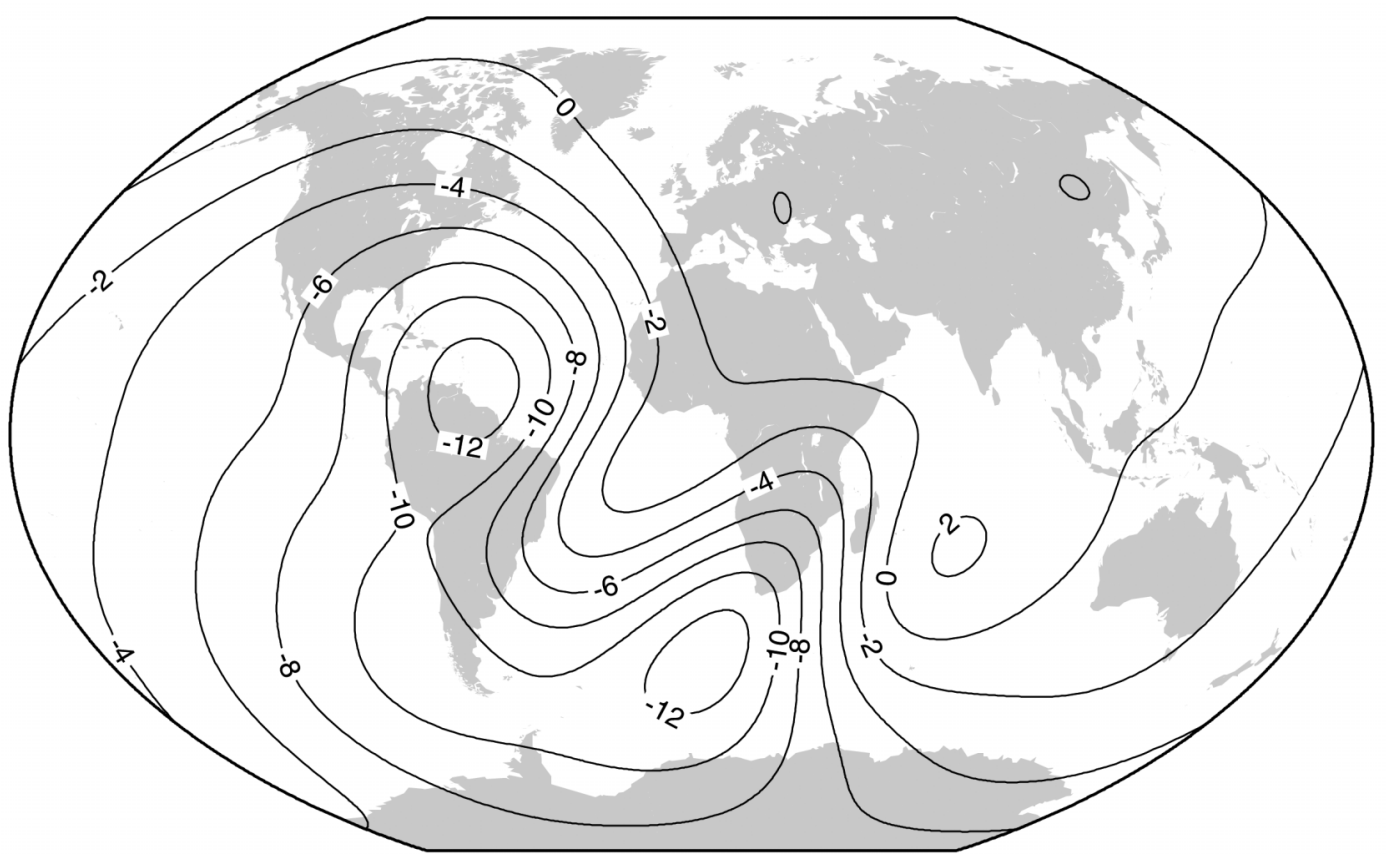

Fig. 3. Change in magnetic field strength $1955.0-2005.0$ as percentage of mean field for same period, from IGRF-10. 


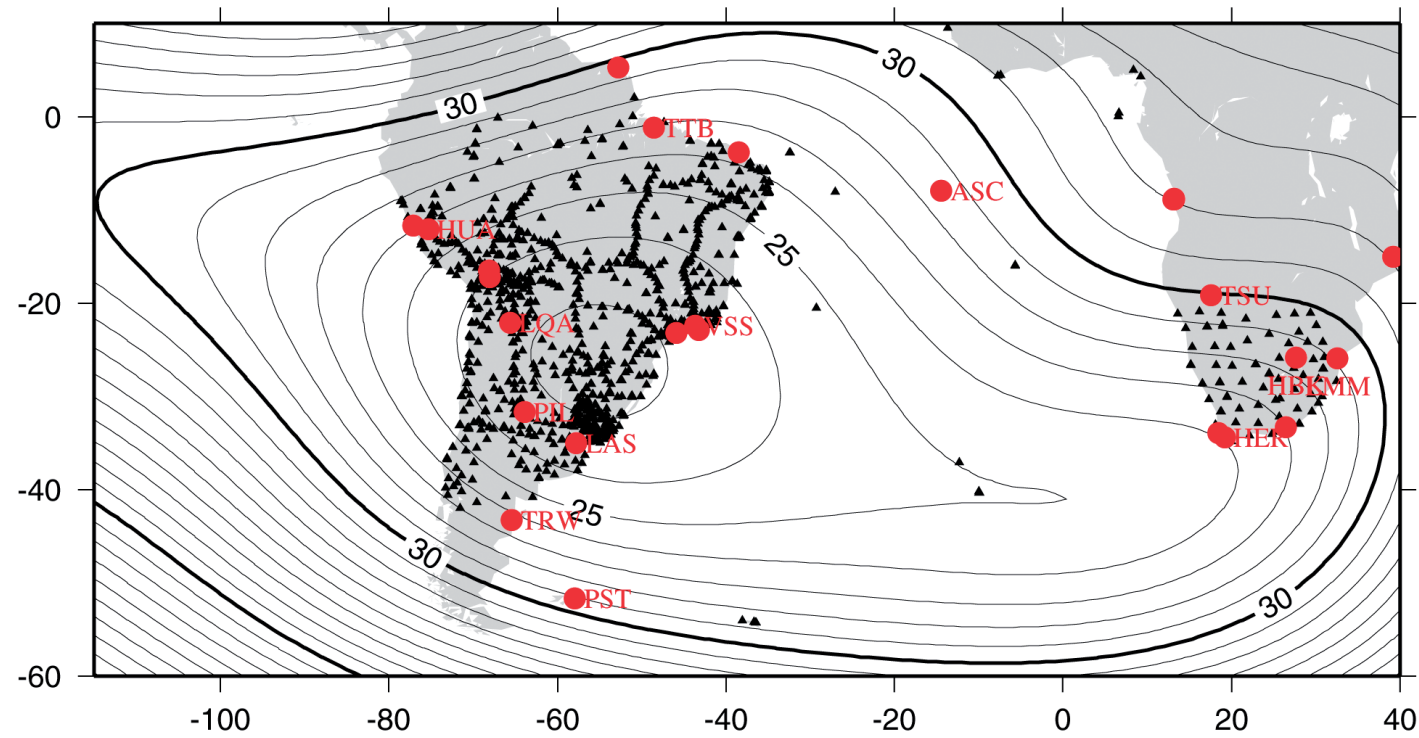

Fig. 4. Locations of observatories (red circles), repeat stations and survey sites (black triangles) where a total intensity of less than $30 \mu \mathrm{T}$ has been observed. Field intensity $(\mu \mathrm{T})$ for 2005.0 from IGRF-10 also shown.

These data are held at the World Data Centre (WDC) for Geomagnetism in Edinburgh. The locations selected are those where a magnetic field strength of less than $30 \mu \mathrm{T}$ has been observed at some time in the past. Along with the polar regions, this region sees many SEUs.

Annual means from observatories in this region with long records are plotted in fig. 5. By using annual means the most significant (i.e. largest amplitude) fields of external origin (storms, daily, seasonal and annual variation) are filtered out: the resulting values mainly represent the field arising from inside the Earth with a very small component attributable to solar cycle effects on fields of external origin. These data would have been used to derive the IGRF depicted in figs. 2-4 but as some of the series extend further back in time we can see that the decrease in the last 50 years is part of a longer-term decrease in the field strength in this part of the world. The sustained, rather high, rate of decrease over South Africa should be noted.

To analyse the irregular external field one needs to use minute mean values or even 1 -sec- ond samples. Minute mean values are available at the WDC for eight of the thirteen observatories shown in fig. 5. One robust, and fairly quick-to-compute, measure of the magnitude of the rapid external field variations is hourly standard deviation. This measure is also widely used in the study of geomagnetically induced currents (GIC) as it is related to the power in the magnetic field variations that drive surface electric fields and GICs (Beamish et al., 2002). However, in order to depict any long-term trends, we take 10-day means (thereby filtering out the diurnal variation) of these (fig. 6).

From fig. 6, the solar cycle signal and the enhanced external field level for Huancayo (HUA) observatory near the dip equator are to be noted. No other obvious long-term trends are apparent.

The same exercise is undertaken for a selection of observatories outwith the SAA area but at similar (geomagnetic) latitudes to those inside. The selected observatories have magnetic field strength more than $30 \mu \mathrm{T}$ but are within the inclination range of those observatories inside the SAA, and, additionally, have series of 

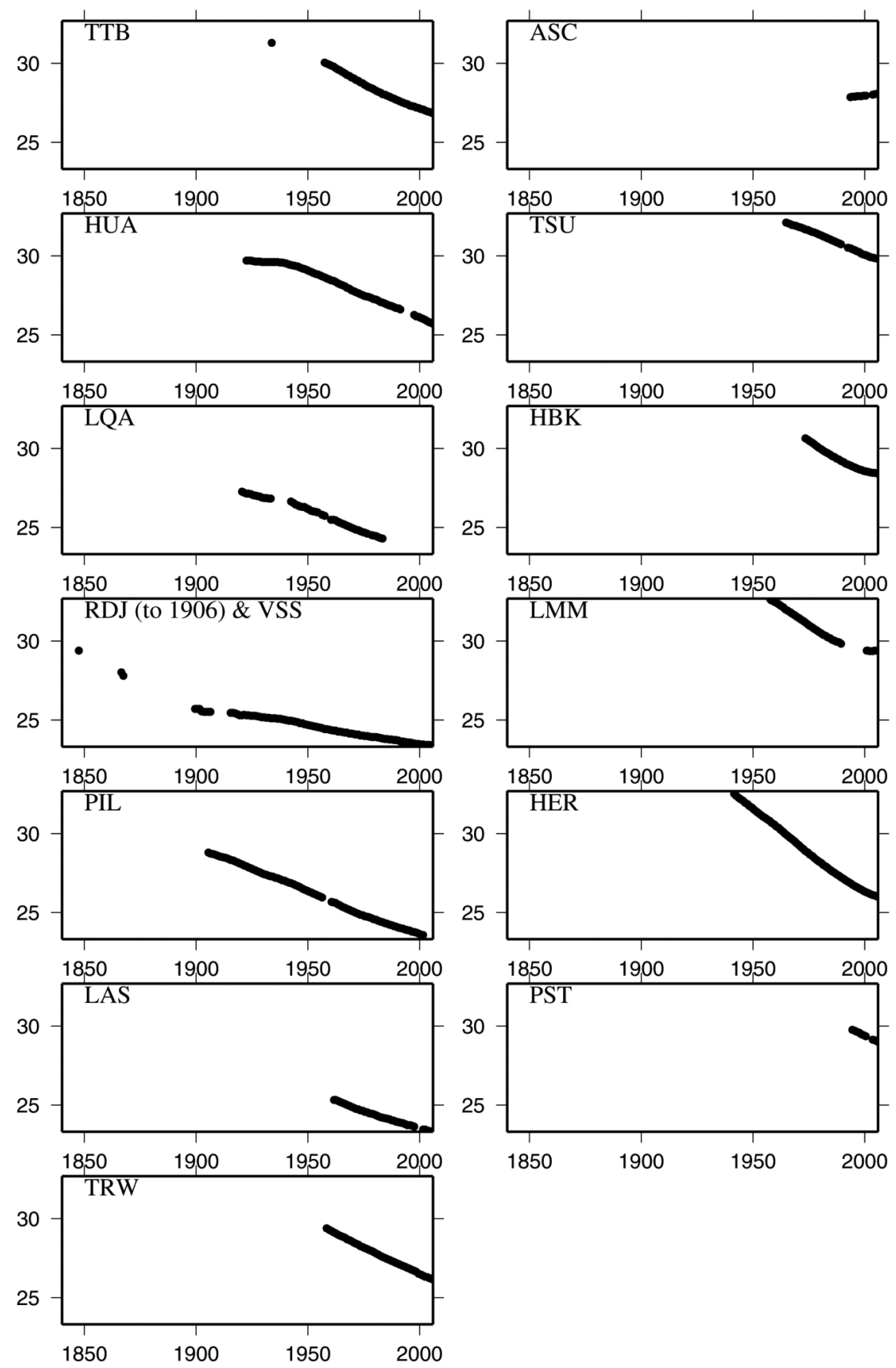

Fig. 5. Annual means of total intensity $(\mu \mathrm{T})$ at selected long-running observatories in region of SAA. Locations of observatories are shown in fig. 4. 

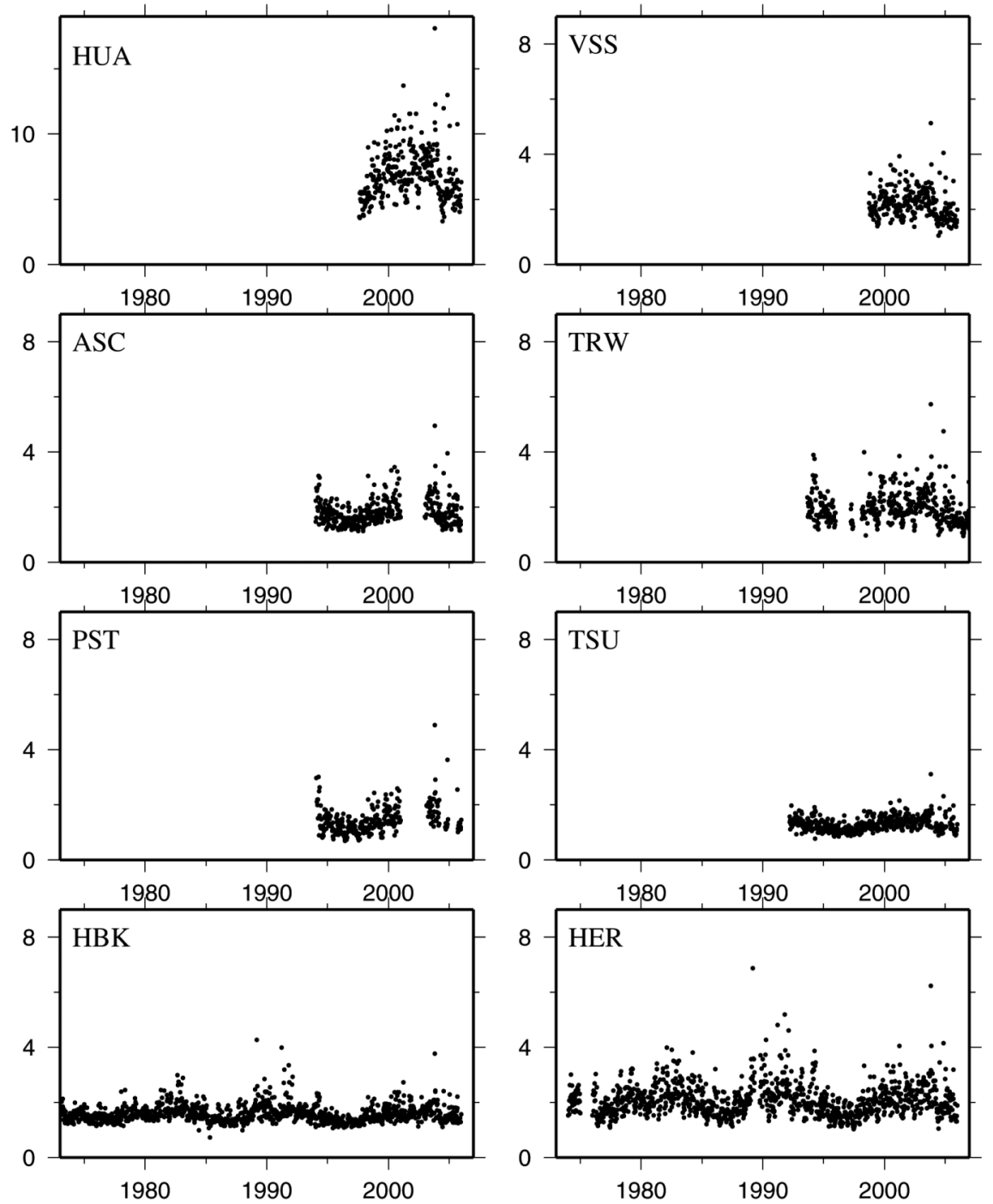

Fig. 6. Ten-day means of hourly standard deviations of total intensity $(\mu \mathrm{T})$. Note different scale used for HUA which is close to dip equator and therefore affected by the equatorial electrojet. Observatories organised by linclination| from left to right, top to bottom.

minute means which extend more than 10 years and have a good global coverage. To demonstrate this selection based on inclination and total intensity, and to illustrate the asymmetry of the Earth's magnetic field introduced by the SAA, fig. 7 shows the locus of modelled values (at 2005.0) of inclination and total intensity for the whole of the Earth's surface and for the observatories used in this study (JCO, LER, ESK and HAD not included in minute mean analysis but are shown on this plot because they are used later for comparing micropulsations).

The effect of high latitude current systems in all selected observatories is assumed to be 


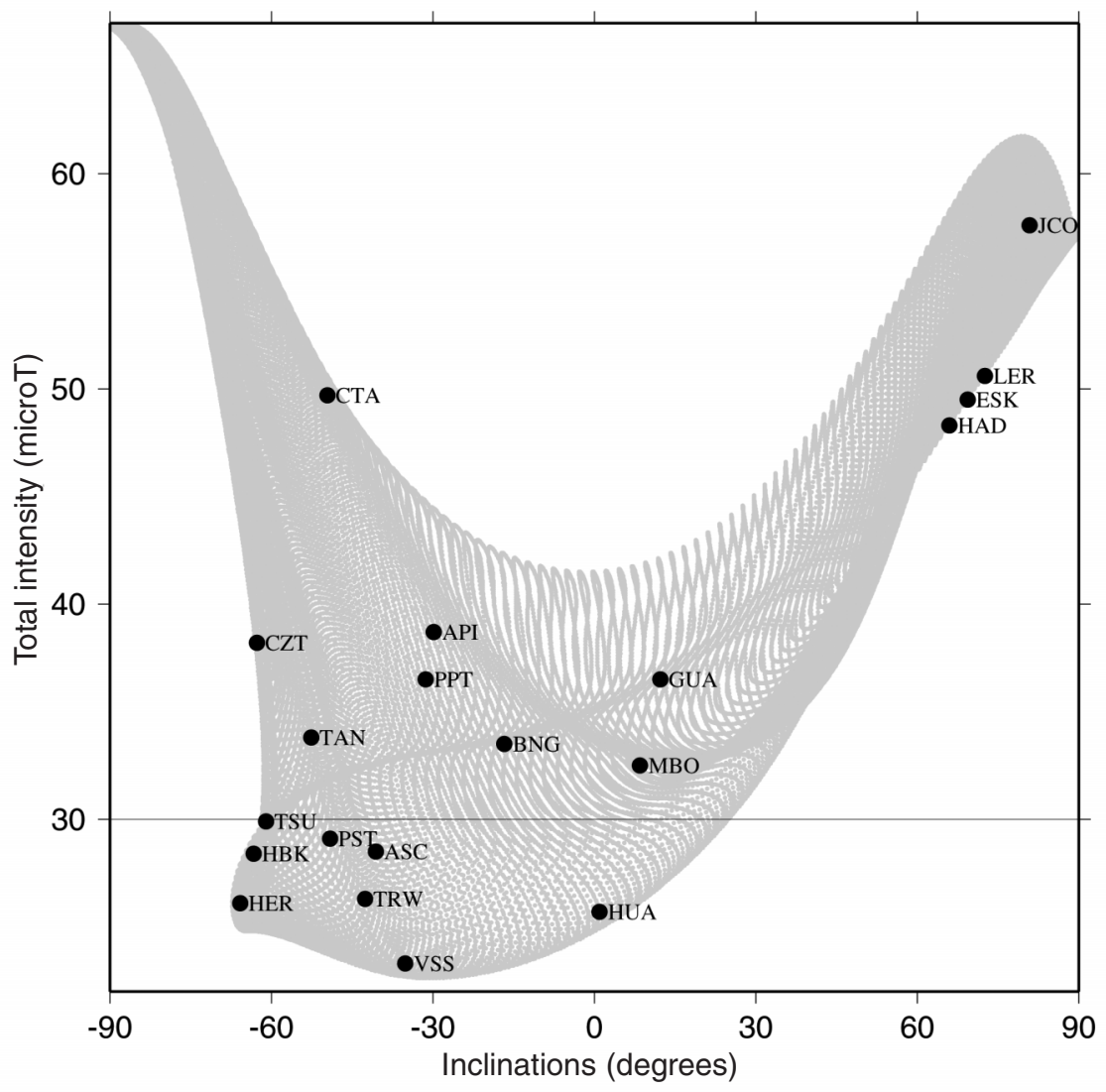

Fig. 7. Locus of modelled values of inclination and total intensity from the 10 th generation IGRF at 2005.0 for the whole of the Earth's surface and for the observatories whose minute means and 1-second values were used in this study. Below the $30 \mu \mathrm{T}$ line indicates region of the SAA. If the field was dipolar the locus would be a parabola

small. Ten-day means of hourly standard deviations are plotted in fig. 8 .

The variable solar cycle amplitude can be seen but without more detailed analysis it is not possible to say whether there is any «local» interaction, for example due to the SAA, between the system generating the external field signals and the internal magnetic field.

Both inclination and strength of the internal field at the observatories used in this study were expected to have an influence on the amplitudes of the external fields observed, but little discernible pattern is observed between the general amplitude of the external fields (as deter- mined by 10-day means of hourly standard deviations) and long-term means of inclination or total intensity.

Finally we take a detailed look at a single magnetic storm and examine its characteristics using 1-second data at several observatories including Ascension and Port Stanley. This is possible because BGS observatories have been routinely collecting 1-second data since 2002. Figure 9 shows the filtered (2-1000 s) north and east components at BGS observatories during the 30th October 2003 «Halloween» magnetic storm. This represents the broadband pulsations. There is a relatively strong $10-20$ minute period 

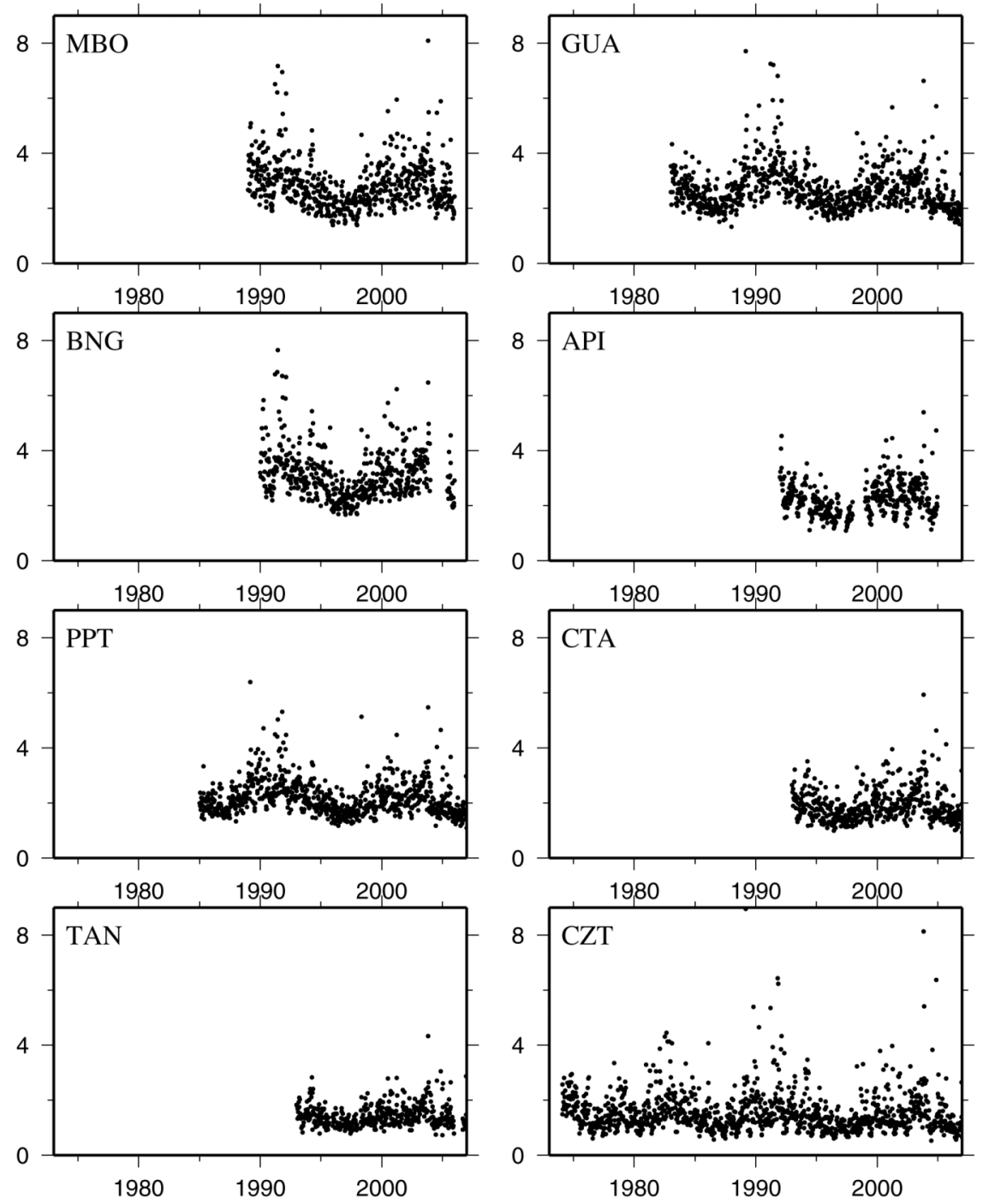

Fig. 8. Ten-day means of hourly standard deviations of $\mathrm{F}(\mu \mathrm{T})$ for observatories outside SAA. Observatories organised by |inclination| from left to right, top to bottom.

oscillation observed at PST between 20:30 and 22:30 that is not as clearly seen elsewhere.

Figure 10 shows filtered (40-150 s) data, representing the $\mathrm{Pi} 2$ pulsation band. Activity in this band occurs in connection with magnetospheric substorms. Note the larger peak amplitude observed at Port Stanley $(\mathrm{L}=1.8)$ compared with Hartland around 20:00 UT, but ab- sence of activity (or at least much reduced activity) from 21:00-23:00 at both PST and ASC, both inside the SAA.

Tables I and II list the RMS and peak amplitudes associated with the pulsations during 30th October 2003 shown in figs. 9 and 10, and compares them with values from a quiet period at same time of day but a few days earlier. 


\section{Broadband ULF Monitor (1-500 mHz: 2-1000 sec)}

Near-Real-Time BGS Magnetic Data Updated Every 10 Minutes.

Up to 23:59UT on 30-10-2003. North (East) Component in Colour (Black)

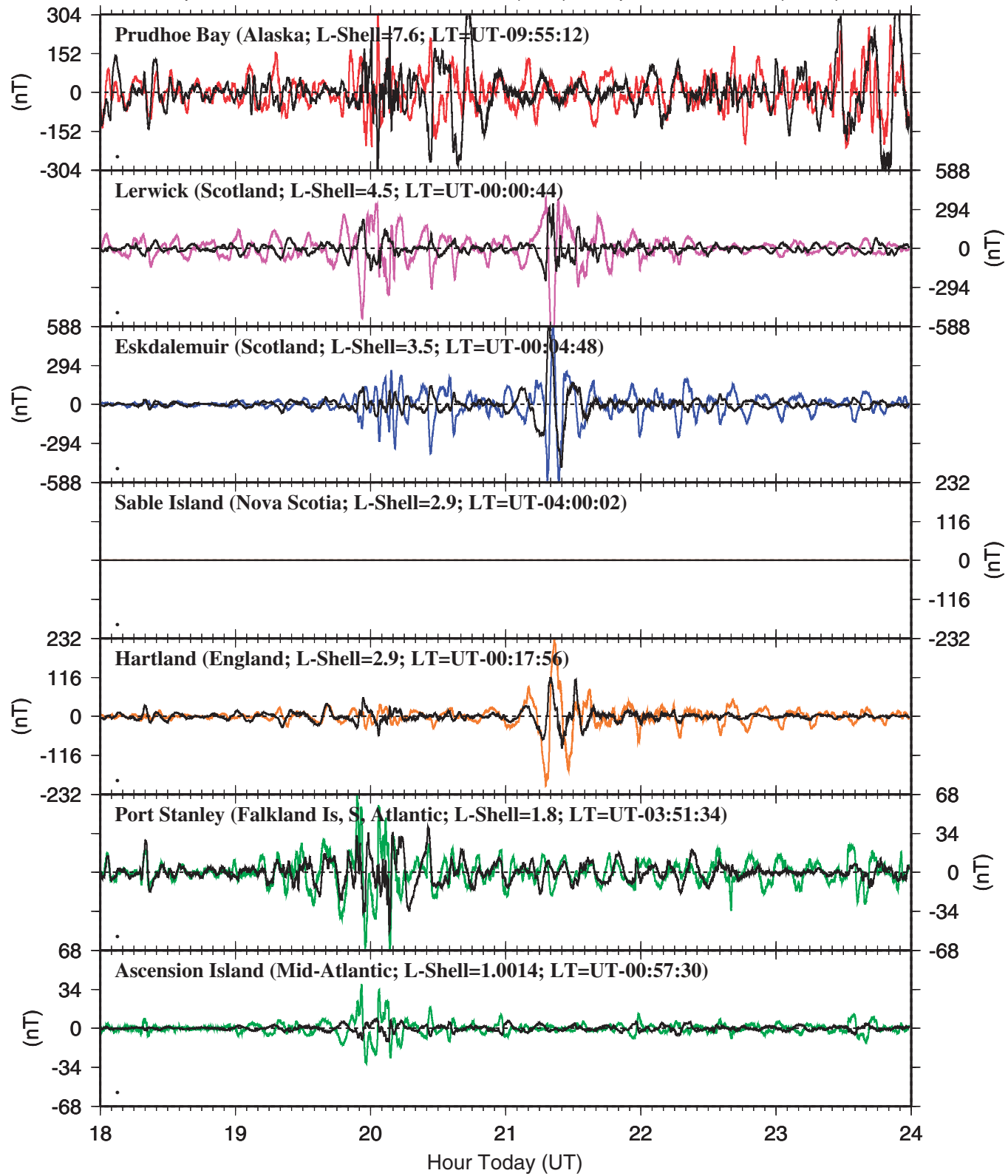

Fig. 9. Stack plot of filtered (2-1000 s) north (X, coloured line) and east (Y, black line) BGS observatory measurements during the 30th October 2003 magnetic storm, for six hours when the 3-hour magnetic activity index $a p$ was sustained at its maximum of 400. Magnetic $L$-shell (McIlwain, 1961) and local time (LT) are noted for each observatory. Note that 1 -second data from the Sable Island observatory were unavailable at this time. 


\section{Pi2 Pulsation Monitor (7-25 mHz: 40-150 sec)}

Near-Real-Time BGS Magnetic Data Updated Every 15 Minutes. Up to 23:59UT on 30-10-2003. North (East) Component in Colour (Black)

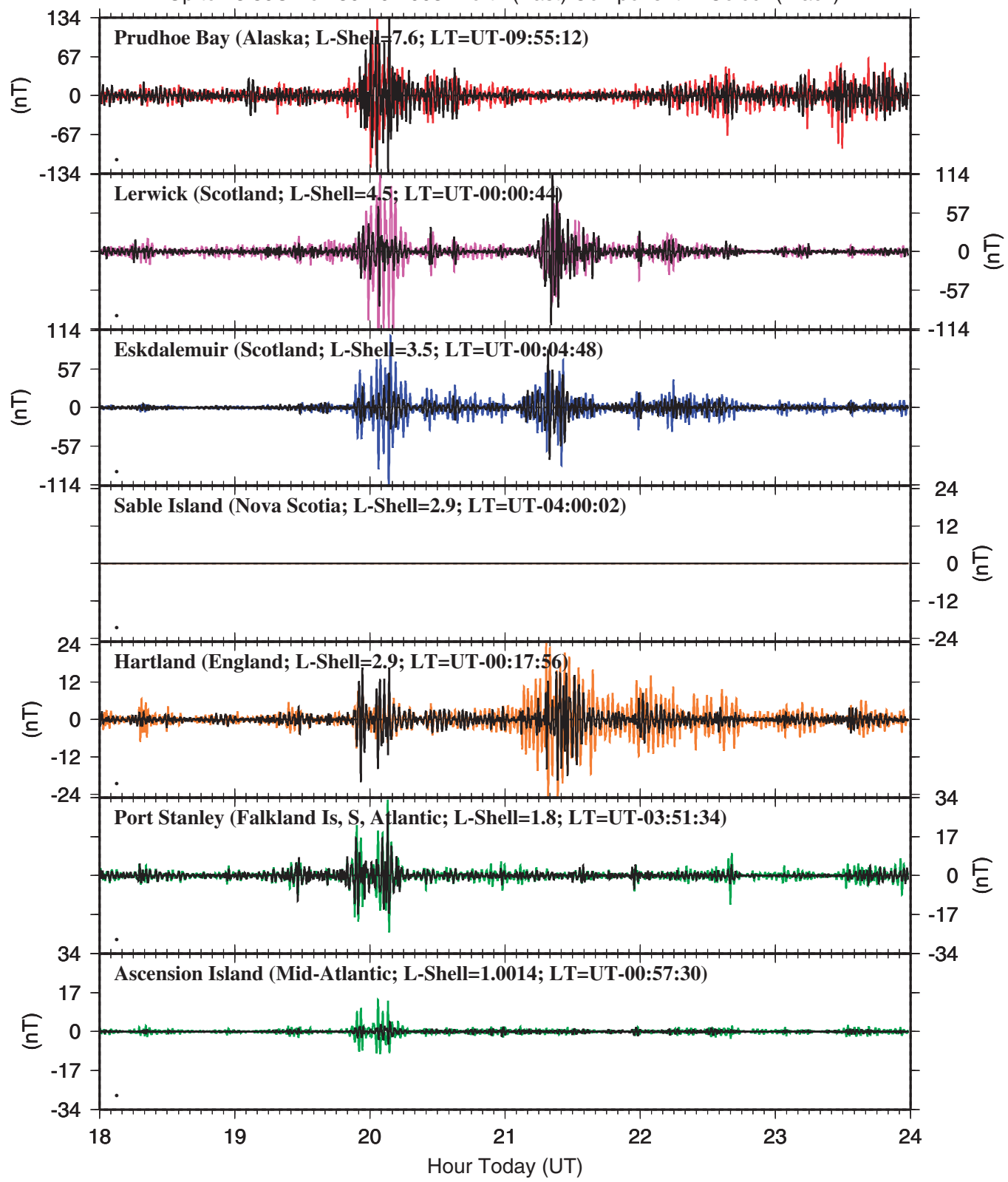

Fig. 10. Similar to fig. 9 but output from the Pi2 waveband (40-150 s). 
Table I. $R M S$ and peak amplitudes for the six observatory filtered series shown in fig. 9 (18:00 - 00:00 UT on 30th October 2003, when $a p=400,400)$, compared to a quiet period from the same month (18:00 - 00:00 UT on 27th October 2003, when $a p=5,4)$. North $(\mathrm{X})$ and east $(\mathrm{Y})$ geocentric amplitudes are given.

\begin{tabular}{ccccccc}
\hline \hline Observatory & L-Shell (Earth Radii) & Activity Conditions & $\begin{array}{c}\text { X rms } \\
(n T)\end{array}$ & $\begin{array}{c}\text { Y rms } \\
(n T)\end{array}$ & $\begin{array}{c}\text { X peak } \\
(n T)\end{array}$ & $\begin{array}{c}\text { Y peak } \\
(n T)\end{array}$ \\
\hline JCO & \multirow{2}{*}{ (.6 } & Storm & 65.8 & 87.8 & 304.4 & 415.5 \\
Alaska & & Quiet & 5.2 & 4.1 & 28.9 & 16.5 \\
LER & 4.5 & Storm & 112.0 & 45.8 & 949.6 & 339.3 \\
Scotland & & Quiet & 0.4 & 0.4 & 2.2 & 2.9 \\
ESK & \multirow{2}{*}{3.5} & Storm & 96.6 & 70.6 & 614.2 & 706.5 \\
Scotland & & Quiet & 0.4 & 0.3 & 2.1 & 1.8 \\
HAD & \multirow{2}{*}{2.9} & Storm & 32.2 & 16.9 & 231.2 & 117.2 \\
England & & Quiet & 0.2 & 0.2 & 1.1 & 0.8 \\
PST & \multirow{2}{*}{1.8} & Storm & 12.5 & 9.0 & 72.7 & 51.7 \\
Falkland Islands & & Quiet & 0.2 & 0.2 & 1.0 & 0.9 \\
ASC & \multirow{2}{*}{1.001} & Storm & 5.6 & 2.3 & 38.6 & 12.1 \\
Ascension & & Quiet & 0.1 & 0.1 & 0.4 & 0.3 \\
\hline
\end{tabular}

Table II. As table I, but showing the RMS and peak amplitudes for the six Pi2 observatory series shown in fig. 10.

\begin{tabular}{ccccccc}
\hline \hline Observatory & L-Shell (Earth Radii) & Activity Conditions & $\begin{array}{c}\text { X rms } \\
(n T)\end{array}$ & $\begin{array}{c}\text { Y rms } \\
(n T)\end{array}$ & $\begin{array}{c}\text { X peak } \\
(n T)\end{array}$ & $\begin{array}{c}\text { Y peak } \\
(n T)\end{array}$ \\
\hline JCO & \multirow{2}{*}{ (n) } & Storm & 16.3 & 16.2 & 133.7 & 173.2 \\
Alaska & & Quiet & 0.6 & 0.4 & 3.4 & 1.8 \\
LER & 4.5 & Storm & 16.1 & 11.0 & 196.4 & 129.3 \\
Scotland & Quiet & 0.1 & 0.1 & 0.8 & 0.3 & \\
ESK & \multirow{2}{*}{3.5} & Storm & 13.6 & 8.1 & 132.1 & 84.0 \\
Scotland & & Quiet & 0.1 & 0.1 & 0.7 & 0.4 \\
HAD & \multirow{2}{*}{2.9} & Storm & 4.2 & 2.8 & 27.6 & 20.1 \\
England & & Quiet & 0.1 & 0.1 & 0.6 & 0.2 \\
PST & \multirow{2}{*}{1.8} & Storm & 3.0 & 2.2 & 33.2 & 25.4 \\
Falkland Islands & & Quiet & 0.1 & 0.1 & 0.2 & 0.1 \\
ASC & \multirow{2}{*}{1.001} & Storm & 1.3 & 0.5 & 14.0 & 5.3 \\
Ascension & & Quiet & 0.1 & 0.1 & 0.2 & 0.1 \\
\hline
\end{tabular}

There is some evidence that SAA stations show more power (shaded in table II) in the Pi2 band $(40-150 \mathrm{~s})$ than might be expected due to their latitude (c.f. equivalent shaded boxes in table I for 2 - $1000 \mathrm{~s}$ ). This provides some sup- port for the result of Trivedi et al. (2005), who claim extra electron precipitation due to SAA causes enhanced pulsation amplitudes. However what is demonstrated here is that this is the case only sporadically. 


\section{Conclusions}

With 15 years experience in operating magnetic observatories on Ascension Island and the Falkland Islands, it is clear that operating a magnetic observatory at a remote location is challenging both in terms of engineering and logistics. It is also costly in time, money and resources, and hence it is not surprising that the distribution of observatories in the South Atlantic is sparse or that data from these observatories sometimes contain gaps. Despite this, the success of the BGS observatories has improved markedly over recent years as the points of failure have been removed and better communications and more reliable instrumentation have been put in place.

The principal factor, however, in successfully maintaining continuous recordings at the BGS observatories has been to have access to local staff with engineering experience. Although the observatories are monitored from the United Kingdom, they have benefited greatly from the help of local engineers who are available to maintain instrumentation should the need arise, as well as to make absolute observations. Also, BGS sites have been selected with a preference for reliable power supply and bi-directional communications, even if this has been at the expense of some data quality. Although this means that the observatories are sited close to other facilities, making use of existing infrastructure to reduce costs, this simplifies installation and improves reliability.

With regards to the hypothesis that the remarkable changes in the internal magnetic field that have been seen in the last few decades in the south Atlantic should have an effect on the magnetic fields generated external to the Earth, we conclude that further analysis is required. At the moment none of the datasets are sufficiently long compared to the 11-year solar cycle to allow isolation of any significant trends. However, the existence of high frequency micropulsation signals at locations where the magnetic field lines close on the Earth far from the polar areas is unexpected. Clearly more study on this is needed using data during several storms. A polarisation study could also be revealing; it may be that there are different $\mathrm{X} / \mathrm{Y}$ phase relationships observed at sites inside and outside the SAA.

\section{Acknowledgements}

We thank all staff involved in the operation of all observatories whose data we have used, and the World Data Centres for Geomagnetism for collation and distribution of data. This paper is published with permission of the Executive Director of BGS (NERC).

\section{REFERENCES}

Beamish, D., T.D.G. Clark, E. Clarke and A.W.P. ThomSON (2002): Geomagnetically induced currents in the UK: geomagnetic variations and surface electric fields, J. Atmos. Solar-Terr. Phys., 64, 1779-1792.

GubBins, D. (2008): Geomagnetic reversals, Nature, 452, 165-167.

Gubbins, D. and J. Bloxham (1985): Geomagnetic field analysis - III. Magnetic fields on the core-mantle boundary, Geophys. J. R. Astron. Soc., 80, 695-713.

HeIRTZleR, J.R. (2002): The future of the South Atlantic anomaly and implications for radiation damage in space, J. Atmos. Solar-Terr. Phys., 64, 1701-1708.

JACKSON, A., A.R.T. JONKERS and M.R. WALKER (2000): Four centuries of geomagnetic secular variation from historical records, Phil. Trans. R. Soc. Lond. A., 358, 957-990.

McIlwain, C.E. (1961): Coordinates for Mapping the Distribution of Magnetically Trapped Particles, J. Geophys. Res., 66, 3681-3691.

Maus, S., S. Macmillan, T. Chernova, S. Choi, D. Dater, V. Golovkov, V. Lesur, F. Lowes, H. LÜHr, W. MaI, S. Mclean, N. Olsen, M. Rother, T. Sabaka, A. ThOMSON, T. Zvereva and InTERNATIONAL AssociaTION OF GEOMAGNETISM, AERONOMY (IAGA), Division V, Working Group VMOD (2005): The 10th generation international geomagnetic reference field, Phys. Earth Planet Int., 151, 320-322.

Trivedi, N.B., P.M. Pathan, Nelson J. Schuch, M. BarRETO and L.G. DUTRA (2005): Geomagnetic phenomena in the South Atlantic anomaly region of Brazil, Adv. Space Res., 36, 2021-2024. 